\title{
Expression of VEGF-A in Intraepithelial and Invasive Cervical Neoplasia
}

Maria Cristina A Westin ${ }^{1}$, Silvia Helena Rabelo-Santos ${ }^{2}$, Liliana A L Ângelo-Andrade ${ }^{3}$, Sophie Derchain ${ }^{3}$, Glauce A Pinto ${ }^{3}$, Sirlei Morais ${ }^{4}$, Paulo L Filho ${ }^{4}$ and Luiz C Zeferino ${ }^{*}$

${ }^{1}$ Department of Obstetrics and Gynecology, State University of Campinas, UNICAMP, School of Medicine, Campinas, Brazil

${ }^{2}$ School of Pharmacy, Federal University of Goiás, Goiânia, Brazil

${ }^{3}$ Department of Pathology, State University of Campinas, UNICAMP, School of Medicine, Campinas, Brazil

${ }^{4}$ Laboratory of Pathology, CAISM- Prof. Dr. Jose Aristodemo Pinotti Women's Hospital, State University of Campinas, UNICAMP, Campinas, Brazil

*Corresponding author: Luiz Carlos Zeferino, Alexander Fleming Street, 101, 13.024-110 Campinas, São Paulo, Brazil, Tel: +55-19 3521 9516; Fax: +55-19 3289 9424; E-mail: zeferino@fcm.unicamp.br

Received date: Aug 29, 2015; Accepted date: Nov 17, 2015; Published date: Nov 18, 2015

Copyright: (c) 2015 Westin MCA, et al. This is an open-access article distributed under the terms of the Creative Commons Attribution License, which permits unrestricted use, distribution, and reproduction in any medium, provided the original author and source are credited.

\begin{abstract}
Angiogenesis seems to be an early event that is associated with disease progression in cervical intraepithelial neoplasia (CIN). The aim of this study was to describe and analyze VEGF-A expression in CIN 3 and invasive cervical carcinoma, in tumor cells and stromal cells. This study comprised three groups: Group 1: 53 cases with CIN 3; Group 2: 24 cases with both CIN 3 and invasive carcinoma components; Group 3: 36 cases with invasive carcinoma. Protein expression was investigated in tumor and stromal cells by immunohistochemistry. Statistical analysis was carried out, considering the mean percentage of immunopositive cells. VEGF-A expression was higher in stromal cells in cases of invasive carcinoma (Group 3) than in CIN 3 cases (Group 1) and this difference was statistically significant. VEGF-A expression showed a borderline association between stromal cells in invasive carcinoma (Group 3) and invasive carcinoma associated with CIN 3 (Group 2). The mean percentage of cells expressing VEGF-A was significantly higher in tumor cells in CIN 3 than in stromal cells in CIN 3 (Group 1). The progression of CIN 3 to invasive carcinoma seems be determined by complex interactions between tumor and stromal cells. These findings reinforce the evidence of the role of stromal cells in tumor invasiveness.
\end{abstract}

Keywords: Angiogenesis inducers; VEGF-A; Uterine cervical neoplasms; Neoplasm invasiveness

\section{Introduction}

Cervical cancer is the third most common cancer in women worldwide and more than $85 \%$ of the global burden occurs in developing countries [1]. Cervical cancer develops from a precancerous lesion referred to as cervical intraepithelial neoplasia (CIN) [2]. Invasion of cervical squamous cell carcinoma begins at the basal cell layer in a field of high-grade cervical intraepithelial neoplasia (CIN 3) [3].

Angiogenesis is the formation of neovasculature from preexisting blood vessels. It is crucial for normal body development and growth. Tumor angiogenesis has been extensively investigated in many human neoplastic conditions. Vascular endothelial growth factor (VEGF) is a potent mitogen responsible for the induction of angiogenesis $[4,5]$. The vascular endothelial growth factor (VEGF) family is comprised of highly conserved secreted glycoproteins that regulate vasculogenesis, hematopoiesis, angiogenesis, lymphangiogenesis and vascular permeability. Four VEGF members have been currently identified and the most important member is VEGF-A (termed VEGF). Other family members are VEGF-B, VEGF-C and VEGF-D. While VEGF-A and VEGF-B are mainly involved in hemangiogenesis, VEGF-C and its close homolog VEGF-D primarily take part in lymphatic vessel growth [6]. In several types of carcinoma, including cervical cancer, a high expression of VEGF has been associated with disease progression and poor outcome [7].
Histologic analysis of premalignant, noninvasive lesions, arising in a variety of organs, has revealed the early tripping of the "angiogenic switch" $[4,7]$. In several types of carcinoma, including cervical cancer, a high VEGF expression has been associated with disease progression, leading to poor patient outcome [8-12]. The term "angiogenic switch" refers to a time-restricted event during tumor progression where the balance between proangiogenic and antiangiogenic factors tilts towards a proangiogenic outcome, resulting in the transition from dormant avascularized hyperplasia to outgrowing vascularized tumor and eventually to malignant tumor progression [7]. It has been indicated that a switch from a lymphangiogenic phenotype towards a hemangiogenic phenotype occurs with invasion, and disease progression in cervical neoplasia. The key molecule involved in this process is VEGF-A, which acts mainly as a potent mitogen on blood endothelial cells via the receptor tyrosine kinase VEGFR-2 [8]. Some studies have reported VEGF expression in premalignant lesions of the uterine cervix [10-12]. Based on clinical and experimental evidence, it was demonstrated that angiogenesis precedes overt tumor formation during chemically induced carcinogenesis $[4,13]$.

Under normal physiological conditions, the stroma acts as an important barrier to epithelial cell transformation. Nevertheless, the stromal compartment undergoes changes in response to emerging epithelial lesions. This compartment has a key role in cancer initiation and progression, including the recruitment of new stromal cells that provide factors involved in cell growth and matrix remodeling [14]. Angiogenesis in cervical carcinogenesis seems to be an early event that occurs in premalignant changes of the cervix. It is associated with disease progression and may serve as a prognostic indicator of cervical squamous cell carcinoma [4]. A better understanding of the angiogenic 
mechanism by which CIN 3 acquires the ability to invade the cervical stroma provides opportunities, e.g. interference with initial tumor development by blocking the "angiogenic switch" which precedes the progression to invasive cancer [8].

Therefore, the aim of this study was to describe and analyze the expression of VEGF-A in CIN 3 and invasive carcinoma of the cervix, in tumor cells and stromal cells.

\section{Materials and Methods}

\section{Ethics statement}

All tissue samples were collected for histologic examination and diagnostic purposes and were thoroughly anonymized for the use in this study. The Institutional Review Board waived the need for written informed consent from the participants. This study was approved by the State University of Campinas Ethics Committee (Approval No. 858/2007 - November 11th, 2007). The manuscript's guarantor affirms that this manuscript is an honest, accurate, and transparent account of the study being reported; that no important aspects of the study have been omitted; and that any discrepancies from the study as planned have been explained [15].

\section{Patients and samples: Case selection}

This comparative observational study of women with cervical neoplasia consisted of three groups. The first group comprised 53 cases of histologic diagnosis of unique CIN 3 (Group 1), the second group comprised 24 cases with both components: CIN 3 (Group 2) and invasive carcinoma (Group 2) and the third group was composed of 36 cases with a histologic diagnosis of invasive carcinoma (Group 3). The study design was constructed assuming that pure CIN 3 (Group 1), CIN 3 associated with invasive carcinoma (Group 2) and pure cervical invasive carcinoma (Group 3) represent progressive steps from CIN 3 to invasive carcinoma.

Cases were selected from records of pathology reports obtained from women consecutively managed in the Women's Hospital, State University of Campinas, Brazil, between 2002 and 2008. Hematoxilineosin paraffin-embedded sections were reviewed and the best representative sample was identified considering tumor tissue and underlying stroma. Specimens were obtained by cervical punch biopsies, conizations or hysterectomies. Despite some limitations of immunohistochemistry analysis of stromal cells, this tissue compartment was analyzed because evidence shows that the stroma plays a relevant role in cervical cancer progression.

\section{Imunohistochemistry assay}

VEGF-A expression was investigated in paraffin-embedded sections using the avidin-biotinperoxidase complex method. Paraffin sections (5 $\mu \mathrm{m}$ thick) were deparaffinized and immersed in 3\% hydrogen peroxidase in methanol to block endogenous peroxidase activity. Next, an antigen retrieval procedure was performed by immersing the slides in $10 \mathrm{mM}$ citrate buffer ( $\mathrm{pH} \mathrm{6.0)}$ and autoclaving these slides at $121^{\circ} \mathrm{C}$ for $10 \mathrm{~min}$. After washing in PBS, the tissue sections were preblocked using 10\% normal goat serum for $15 \mathrm{~min}$. The protocol for the Dako LSAB 2 peroxidase kit (Dako, Kyoto, Japan) was followed. The sections were incubated overnight with primary antibodies in a humidity chamber at $4^{\circ} \mathrm{C}$.
Primary antibodies used for this study were polyclonal mouse antihuman VEGF (DaKo). The working dilutions of primary antibodies against VEGF-A were 1:100. Sections were rinsed with PBS for $15 \mathrm{~min}$ and incubated for $1 \mathrm{~h}$ with the secondary antibody (biotinylated goat anti-mouse and rabbit immunoglobulin G secondary antibody; Dako). The sections were then incubated with streptavidin-peroxidase complex, using 3,3'-diaminobenzidine as a chromogen. Sections were counterstained with Mayer's hematoxylin. Specificity of the immunohistochemical reactions was checked by omitting the primary antibody.

The methodology for interpretation of immunostaining sections consisted in the following steps:

1 - Identification of representative regions of lesions selected according to group (CIN 3, invasive carcinoma and underlying stromal lesion). Selection criteria for these representative regions were: image sharpness, areas of higher intensity of cellular immunoreaction, a similar proportion of stromal and tumor regions in the same picture, with the exclusion of necrotic regions. Stromal regions with a high concentration of inflammatory cells were excluded.

2 - Images were captured in these regions under a magnification of 400X. In groups of CIN 3 and invasive carcinoma, from one to eight pictures were taken. In Group 2 which included cases of invasive cell carcinoma associated with CIN 3, pictures were taken separately.

3 - Selection of one photograph per lesion and its underlying stroma was made for quantitative and qualitative analysis of the immunohistochemical reaction.

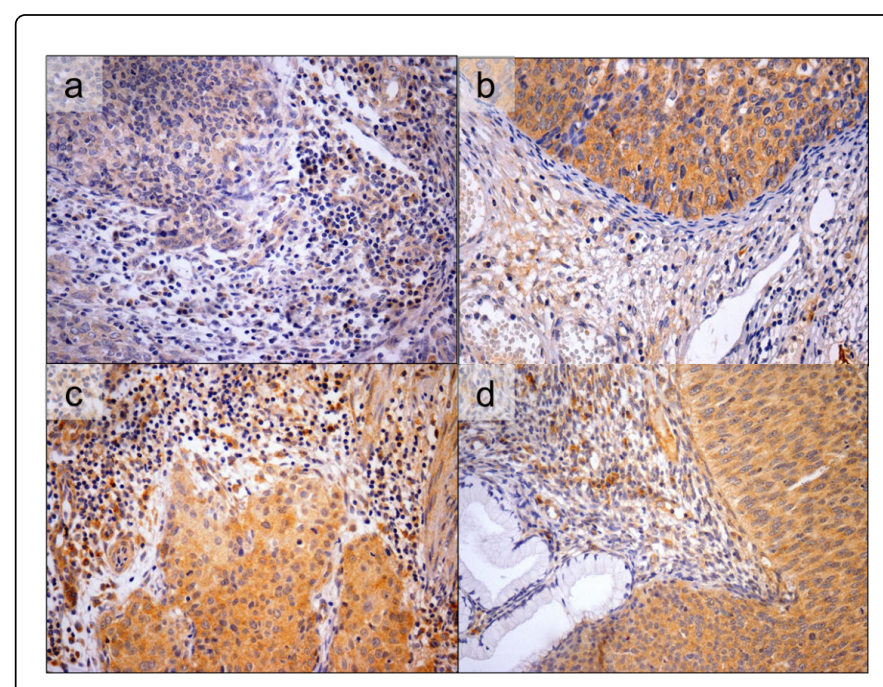

Figure 1: Percentage of tumor and stromal cells expressing VEGF-A. A: Invasive carcinoma (Group 3): tumor cells: $61,8 \%$, stromal cells: 75,7\%; B: CIN 3 (Group 1): tumor cells: 70,2\%, stromal cells: 53,9\%; C: Invasive carcinoma compartment (Group 2): tumor cells: $91,3 \%$, stromal cells: 96,7\%; D: CIN 3 compartment (Group 2): tumor cells: $65,9 \%$, stromal cells: $60,9 \%$.

4 - Calculation of total cell number and number of immunostained cells (stromal cells and tumor cells, separately) was made by two observers using morphometric software (Image Pro Plus ${ }^{\oplus}$, version 6.3, Olympus) (Figure 1). To assess the percentage of immunostained cells, at least 1,000 tumor cells and stromal cells per case were counted. 
Page 3 of 5

\section{Statistical analysis}

Statistical analysis was carried out, considering the percentages of immunopositive tumor cells and stromal cells within each group and between diagnostic groups. The CIN 3 and invasive carcinoma components from group 2 were analyzed separately. Analyses of the percentages of tumor cells and stromal cells between two diagnostic groups were carried out using the Mann-Whitney and Wilcoxon Signed Rank tests. A comparison of the mean percentage of tumor cells and stromal cells within each diagnostic group was made by using the paired t-test. The reason for this was because although the percentages of immunopositive tumor cells and stromal cells did not show a normal distribution, the differences between them were normally distributed. Differences were considered significant when $\mathrm{p}$ value was less than 0.05 .

\section{Results}

VEGF-A expression was observed in the cytoplasm of tumor cells and surrounding stroma in all analyzed cases. The mean percentage of cells expressing VEGF-A was significantly higher $(\mathrm{p}=0.0030)$ in tumor cells $($ mean $=54.1 \% ; \mathrm{SD}=25.9)$ than in stromal cells (mean $=43.7 \%$; $\mathrm{SD}=26.1$ ) in cases diagnosed as CIN 3 (Group 1) (Table 1). In invasive carcinoma (Group 3), the mean percentage of cells expressing VEGF-A was higher in stromal cells than in tumor cells (mean $=48.1 \%$; $\mathrm{SD}=28.8)$, but the difference was not statistically significant $(\mathrm{p}=0.0840)$ (Table 1). There was no statistically significant difference between the mean percentage of cells expressing VEGF-A in both tumor cells and stromal cells in CIN 3 and invasive carcinoma components from Group 2 (Table 1).

\begin{tabular}{|c|c|c|c|c|c|c|c|c|c|}
\hline \multicolumn{10}{|c|}{ CIN 3 (Group 1) } \\
\hline Cells & $\mathbf{n}$ & Mean & SD & Min. & Q1 & Median & Q3 & Max. & p-value \\
\hline Tumor & 53 & 54.1 & 25.9 & 5.6 & 35.6 & 54.2 & 76.7 & 97.9 & \multirow[t]{2}{*}{0.0030} \\
\hline Stromal & 53 & 43.7 & 26.1 & 2.5 & 20.2 & 40.1 & 59.8 & 98.5 & \\
\hline \multicolumn{10}{|c|}{ CIN 3 (Group 2) } \\
\hline Tumor & 24 & 53.0 & 29.5 & 1.0 & 26.1 & 59.4 & 71.3 & 96.2 & \multirow[t]{2}{*}{0.7218} \\
\hline Stromal & 24 & 50.8 & 25.6 & 1.4 & 34.6 & 49.5 & 69.2 & 94.6 & \\
\hline \multicolumn{10}{|c|}{ Invasive Carcinoma (Group 2) } \\
\hline Tumor & 24 & 45.9 & 28.8 & 1.3 & 20.3 & 48.8 & 65.9 & 96.1 & \multirow[t]{2}{*}{0.6080} \\
\hline Stromal & 24 & 42.8 & 27.4 & 0.2 & 23.6 & 38.7 & 62.3 & 96.7 & \\
\hline \multicolumn{10}{|c|}{ Invasive Carcinoma (Group 3) } \\
\hline Tumor & 36 & 48.1 & 28.8 & 0.7 & 21.1 & 45.1 & 69.4 & 97.1 & \multirow[t]{2}{*}{0.0840} \\
\hline Stromal & 36 & 56.5 & 23.5 & 3.6 & 43.3 & 59.1 & 72.2 & 96.4 & \\
\hline
\end{tabular}

Table 1: Comparison of mean percentages of tumor and stromal cells VEGF-A immunopositive in each diagnostic group.

The percentage of stromal cells expressing VEGF-A was significantly higher $(\mathrm{p}=0.01$ ) in invasive carcinoma (Group 3) than in CIN 3 (Group 1). The percentage of stromal cells expressing VEGF-A in invasive carcinoma (Group 3) was higher than in the invasive carcinoma component from Group 2, and this difference was statistically significant $(\mathrm{p}=0.0573)$. There was no statistically significant difference in the percentage of cells expressing VEGF-A in both tumor cells and stromal cells among: a) CIN 3 and invasive carcinoma components in Group 2, b) between CIN 3 (Group 1) and CIN 3 (Group 2) and c) invasive carcinoma (Group 3) and invasive carcinoma component (Group 2) (Figure 2).

\section{Discussion}

This study showed that VEGF-A expression is higher in tumor cells than in stromal cells in CIN 3. However, in cervical cancer cases, stromal cells show a higher VEGF-A expression than tumor cells. Similarly, VEGF-A expression is higher in stromal cells in invasive carcinoma than in CIN 3. All cancers contain a diverse population of cells, including those harboring genetic mutations typically referred to as "tumor" or "cancer" cells as well as other cell types that are activated and/or recruited to the local microenvironment, e.g., fibroblasts, innate and adaptive immune cells, and cells that line blood and lymphatic vessels. Reciprocal interactions between these responding "normal" cells, their mediators, structural components of the ECM, and genetically altered neoplastic cells regulate all aspects of tumorigenicity [14].

There is increasing evidence to support the understanding that tumor cells and stromal cells contribute to tumor mass formation and characteristics $[7,16]$. Based on clinical and experimental research, it has been demonstrated that tumor angiogenesis is dependent on stromal cell action and precedes overt tumor formation during chemically induced carcinogenesis $[4,13]$.

High VEGF levels in the tumor microenvironment and consequent activation of a signalling pathway stimulate the growth and migration of lymphatic and blood endothelial cells, thereby promoting vascular invasion by tumor cells [17]. Tumor vasculature is dependent not only on endothelial cells but also other stromal cells, e.g. pericytes. Pericytes are affected by factors secreted by tumor cells. There is a loose association between tumor pericytes and endothelial cells which could affect their survival, as well as contribute to the presence of intercellular gap junctions or openings. These gaps could provide 
Citation: Westin MCA, Rabelo-Santos SH, Ângelo-Andrade LAL, Derchain S, Pinto GA, et al. (2015) Expression of VEGF-A in Intraepithelial and Invasive Cervical Neoplasia. J Cytol Histol S3: 022. doi:10.4172/2157-7099.S3-022

Page 4 of 5

tumor cells with relatively easy access to the intravascular space $[18,19]$.

Inflammatory cells also regulate endothelial cell functions related to tumor angiogenesis. Tumor cells are surrounded by an infiltrate of inflammatory cells, lymphocytes, neutrophils, macrophages and mast cells. These cells communicate through a complex network of intercellular signaling pathways, mediated by surface adhesion molecules, cytokines and VEGF expression. Therefore, inflammatory cells cooperate and synergize not only with stromal cells, but also malignant cells to stimulate endothelial cell proliferation and blood vessel formation $[4,20]$.

\section{VEGF-A: tumor cells}

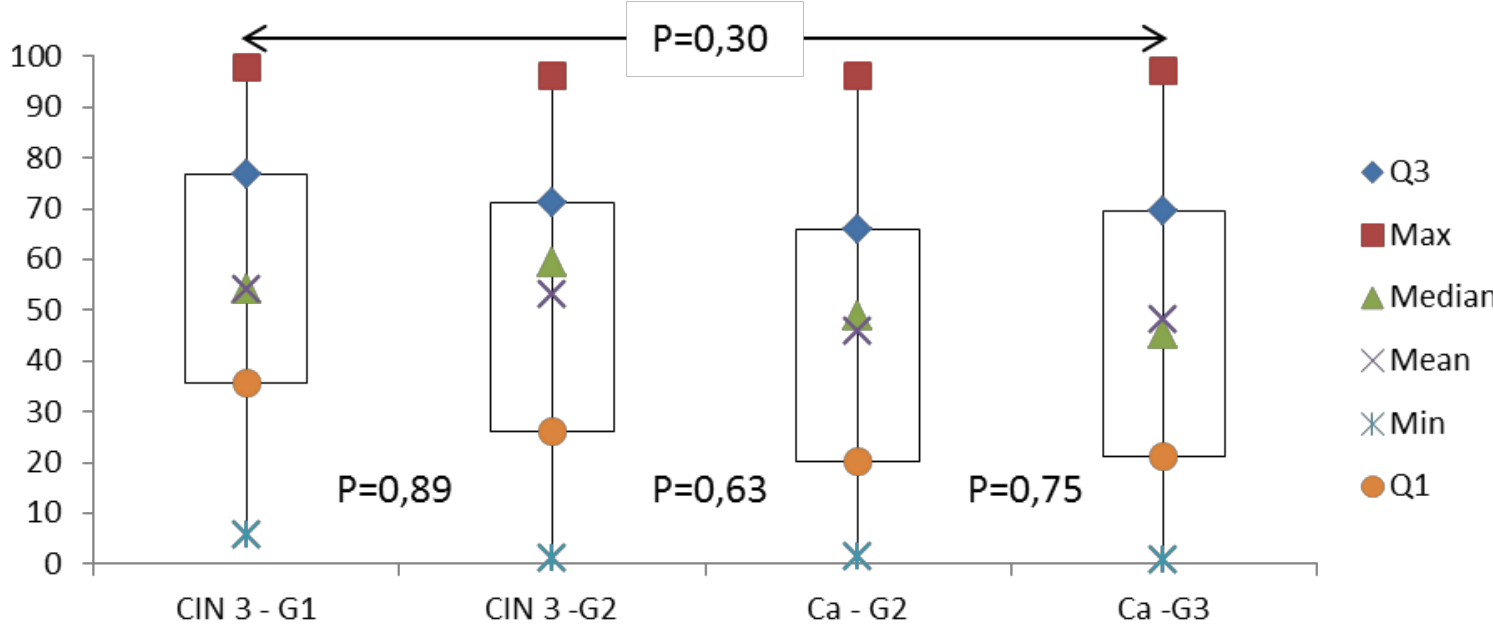

\section{VEGF-A: stromal cells}

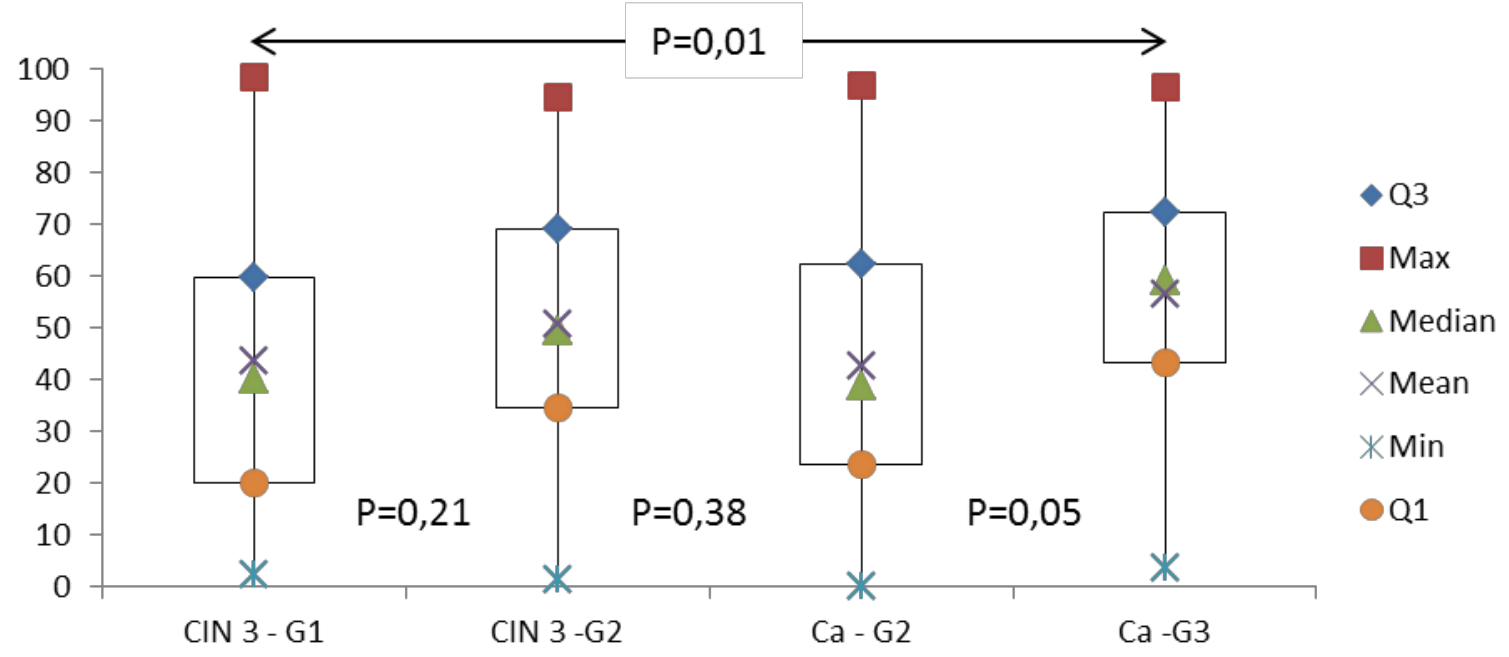

Figure 2: VEGF-A expression in Cervical Neoplasia. CIN 3: Cervical intraepithelial neoplasia grade 3; CA: Invasive carcinoma, G1 to G3: Group 1 to Group 3, Min: minimum, Max: maximum, Q1: $1^{\circ}$ percentile (25\%), Q3: $3^{\circ}$ percentile (75\%). In CIN 3, VEGF-A expression was higher in tumor cells than in stromal cells. In a comparison between invasive carcinoma and CIN 3, VEGF-A expression showed no change in tumor cells, but increased significantly in stromal cells.

In cervical carcinogenesis, angiogenesis follows the current understanding of this issue. Angiogenesis occurs in premalignant changes of the cervix and is associated with disease progression $[4,21]$. Some studies have reported VEGF-A expression in the stage of premalignant lesions of the uterine cervix [10-12]. Hammes et al. [12] showed that stromal VEGF expression was observed in 53.3\% and $75.0 \%$ cases of CIN 3 and invasive carcinoma, respectively. According to these authors, VEGF-A could be a factor in the development and progression of CIN lesions and a potential molecular target for studies 
Citation: Westin MCA, Rabelo-Santos SH, Ângelo-Andrade LAL, Derchain S, Pinto GA, et al. (2015) Expression of VEGF-A in Intraepithelial and Invasive Cervical Neoplasia. J Cytol Histol S3: 022. doi:10.4172/2157-7099.S3-022

Page 5 of 5

of cervical cancer prevention and treatment. Stepan et al. [22] observed that VEGF-A is characteristic of cervical tumor angiogenesis in both early and advanced tumor stages.

Limitations of this study were failure to use normal tissue as control and the subjective nature of immunostaining in the evaluation of histologic samples. Cancers are heterogeneous cellular entities whose growth is dependent upon reciprocal interactions between genetically altered "initiated" cells and the dynamic microenvironment in which they live [14]. VEGF-A expression was higher in tumor cells than in stromal cells. In a comparison between invasive carcinoma and CIN 3, VEGF-A expression showed no change in tumor cells, but increased significantly in stromal cells. The progression of CIN 3 to invasive carcinoma seems be determined by complex interactions between tumor and stromal cells. These findings reinforce the evidence of the role of stromal cells in tumor invasiveness and can help in understanding the prognosis of cervical neoplasia.

\section{Acknowledgments}

We acknowledge the support and assistance of the Department of Pathology, State University of Campinas, UNICAMP in obtaining the tissues for this project.

\section{Funding}

This work was supported by the Fundação de Amparo à Pesquisa do Estado de São Paulo - FAPESP. Grant number: 2007/54709-0.

\section{References}

1. http://globocan.iarc.fr/

2. Tiltman AJ (2005) The pathology of cervical tumours. Best Pract Res Clin Obstet Gynaecol 19: 485-500.

3. Reich O, Pickel H, Tamussino K, Winter R (2001) Microinvasive carcinoma of the cervix: site of first focus of invasion. Obstet Gynecol 97: 890-892.

4. Raica M, Cimpean AM, Ribatti D (2009) Angiogenesis in pre-malignant conditions. Eur J Cancer 45: 1924-1934.

5. Delli CJ, Karam AK, Montgomery L (2010) Vascular endothelial growth factor and its relationship to the prognosis and treatment of breast, ovarian, and cervical cancer. Angiogenesis 13: 43-58.

6. Van TPO, Steele D, Lowe DG, Baithun S, Beasley N, et al. (2003) Expression of vascular endothelial growth factor (VEGF)-C and VEGF-D, and their receptor VEGFR-3, during different stages of cervical carcinogenesis. J Pathol 201: 544-554.

7. Hanahan D, Weinberg RA (2011) Hallmarks of cancer: The next generation. Cell 144: 646-674.

8. Baeriswyl V, Christofori G (2009) The angiogenic switch in carcinogenesis. Semin Cancer Biol 19: 329-337.

9. Kuemmel S, Thomas A, Landt S, Fuger A, Schmid P, et al. (2009) Circulating vascular endothelial growth factors and their soluble receptors in pre-invasive, invasive and recurrent cervical cancer. Anticancer Res 29: 641-645.

10. McCune SKK, Weidner N (1994) Demonstration and characterization of the angiogenic properties of cervical dysplasia. Cancer Res 54: 800-804.

11. Baritaki S, Sifakis S, Huerta-Yepez S, Neonakis IK, Soufla G, et al. (2007) Overexpression of VEGF and TGF-beta1 mRNA in Pap smears correlates with progression of cervical intraepithelial neoplasia to cancer: Implication of YY1 in cervical tumorigenesis and HPV infection. Int J Oncol 31: 69-79.

12. Hammes LS, Tekmal RR, Naud P, Edelweiss MI, Kirma N, et al. (2008) Up-regulation of VEGF, c-fms and COX-2 expression correlates with severity of cervical cancer precursor (CIN) lesions and invasive disease. Gynecol Oncol 110: 445-451.

13. Hanahan D, Folkman J (1996) Patterns and emerging mechanisms of the angiogenic switch during tumorigenesis. Cell 86: 353-364.

14. Tlsty TD, Coussens LM (2006) Tumor stroma and regulation of cancer development. Annu Rev Pathol 1: 119-150.

15. Altman DG, Moher D (2013) Declaration of transparency for each research article. BMJ 347: 44796 .

16. Hanahan D, Weinberg RA (2000) The hallmarks of cancer. Cell 100: 57-70.

17. Moserle L, Casanovas O (2013) Anti-angiogenesis and metastasis: A tumour and stromal cell alliance. J Intern Med 273: 128-137.

18. Gerhardt H, Semb H (2008) Pericytes: Gatekeepers in tumour cell metastasis? J Mol Med (Berl) 86: 135-144.

19. Xian X, Håkansson J, Ståhlberg A, Lindblom P, Betsholtz C, et al. (2006) Pericytes limit tumor cell metastasis. J Clin Invest 116: 642-651.

20. Bergers G, Hanahan D (2008) Modes of resistance to anti-angiogenic therapy. Nat Rev Cancer 8: 592-603.

21. Dai Y, Zhang X, Peng Y, Wang Z (2005) The expression of cyclooxygenase- VEGF and PGs in CIN and cervical carcinoma. Gynecol Oncol 97: 96-103.

22. Stepan D, Simionescu C, Stepan A, Muntean M, Voinea B (2012) VEGF and CD105 immunoexpression in squamous cervical carcinomas and associated precancerous lesions. Rom J Morphol Embryol 53: 585-589.
This article was originally published in a special issue, entitled:

"Cytopathology", Edited by Borislav A. Alexiev, University of Maryland Medical Center, USA 